\title{
VARIAÇÕES NO TEOR E NA COMPOSIÇÃO VOLÁTIL DE Hyptis marrubioides EPL. CULTIVADA NO CAMPO E EM CASA DE VEGETAÇÃO
}

\author{
Priscila Pereira Botrel*, José Eduardo Brasil P. Pinto, Ana Clara Caxito de Araújo e Suzan Kelly Vilela Bertolucci \\ Departamento de Agricultura, Universidade Federal de Lavras, CP 3037, 37200-000 Lavras - MG, Brasil \\ Felipe Campos Figueiredo \\ Departamento de Ciência do Solo, Universidade Federal de Lavras, CP 3037, 37200-000 Lavras - MG, Brasil \\ Pedro Henrique Ferri e Deomar Plácido da Costa \\ Instituto de Química, Universidade Federal de Goiás, CP 131 Goiânia - GO, Brasil
}

Recebido em 10/10/08; aceito em 10/6/09; publicado na web em 25/11/09

\begin{abstract}
VARIATION IN THE CONTENT AND VOLATILE COMPOSITION OF Hyptis marrubioides Epl. CULTIVATED IN FIELD AND GREENHOUSE. This work describes the chemical composition of the volatile oil of Hyptis marrubioides cultivated in field and greenhouse. The experimental design was completely randomized, with ten replications for each type of cultivation. The volatile oil was extracted by hydrodistillation and analyzed by GC-MS. The highest content of volatile oil was found for plants grown in field. The highest percentage of the compounds present in oils was observed in samples grown in the field, such as germacra-4(15),5,10(14)trien-1- $\alpha$-ol (16.34\%), $\beta$-caryophyllene (10.42\%), $\gamma$-muurolene (12.83\%) and trans-thujone $(9.98 \%)$. However, some compounds were found only in plants grown in a greenhouse, such as cis-muurol-5-en-4 $\alpha$-ol (10.84\%), $\alpha$-cadinol (3.06\%) and eudesma-4(15),7dien-1 $\beta$-ol $(6.82 \%)$.
\end{abstract}

Keywords: Hyptis marrubioides; Lamiaceae; volatile chemical composition.

\section{INTRODUÇÃO}

O gênero Hyptis apresenta uma grande diversidade morfológica, principalmente na região do cerrado brasileiro, com cerca de 300 a 400 espécies. ${ }^{1}$ Apresentam um aroma característico, sendo algumas espécies utilizadas no tratamento de infecções gastrointestinais, câimbras, dores e no tratamento de infecções de pele. ${ }^{2}$

H. marrubioides Epl., pertencente à família Lamiaceae, é denominada vulgarmente hortelã do campo. As Lamiaceae pertencem à ordem Tubiflorae (Lamiales), abrangendo cerca de 200 gêneros e aproximadamente 3.200 espécies distribuídas em todo o mundo.

Estudos demonstraram algumas atividades biológicas importantes para este gênero, tais como atividades antifúngica, ${ }^{3}$ antibacteriana, ${ }^{4}$ antiulcerogênica, ${ }^{5}$ larvicida, ${ }^{6}$ antidepressiva. ${ }^{7}$

Os principais constituintes químicos encontrados em H. marrubioides, ao se estudar a variabilidade química presente no óleo volátil desta espécie em duas localidades da região do cerrado brasileiro, foram cariofila-4(14),8(15)-dien-5 $\beta$-ol, eudesma-4(15),7-dien-1 $\beta$-ol, óxido de cariofileno e $(\beta)$-cariofileno. ${ }^{8}$

A composição dos metabólitos secundários nas plantas é resultado do balanço entre biossíntese e transformações que ocorrem durante o crescimento, em decorrência principalmente de fatores genéticos, ambientais e do manejo agronômico utilizado. ${ }^{9}$ Fatores ambientais têm grande influência sobre o desenvolvimento de plantas; particularmente para plantas aromáticas, tais fatores influenciam sobremaneira no teor e na composição química de óleos essenciais, ${ }^{10}$ assim como a técnica de cultivo. ${ }^{11}$

Para a espécie H. marrubioides não existem trabalhos relacionando a composição química com a condição de cultivo. Desta forma, o presente trabalho objetivou avaliar a composição química e o teor do óleo volátil de $H$. marrubioides em plantas cultivadas em condição de campo e casa de vegetação.

*e-mail: botrelpp@bol.com.br

\section{PARTE EXPERIMENTAL}

\section{Condições de cultivo}

O cultivo das plantas em campo foi realizado na Fazenda Experimental Gota da Esperança localizada na Universidade Federal de Lavras, UFLA. O município de Lavras localiza-se na região Sul de Minas Gerais. Situa-se a $21^{\circ} 14^{\prime}$ de Latitude Sul e $45^{\circ} 00^{\prime}$ de Longitude Oeste de Greenwich a uma altitude de $918 \mathrm{~m}$. Na Tabela 1 encontram-se os dados climatológicos referentes ao dia de coleta das folhas.

Plântulas de $H$. marrubioides cultivadas in vitro foram transplantadas para bandejas contendo substrato Plantmax ${ }^{\circledR}$, onde permaneceram por 45 dias sob irrigação em casa de vegetação aclimatizada. $\mathrm{O}$ transplantio das 100 mudas para o campo foi realizado em 15 de fevereiro de 2007. O plantio foi realizado em covas, espaçamento 1,5 x 1,0 m totalizando 40 mudas. Foram realizadas duas adubações, uma com 30 e 60 dias após o plantio, $10 \mathrm{~g} /$ cova de NPK (20-0-20). A colheita de folhas in natura foi realizada 455 dias após o transplantio das mudas, próximo às 8:00 h da manhã. Coletaram-se apenas folhas do terço superior da planta. Para cada repetição, que foi representada por três plantas colhidas aleatoriamente, utilizou-se uma amostra composta de $40 \mathrm{~g}$ de massa fresca para a extração do óleo volátil.

O cultivo das plantas em casa de vegetação foi realizado no Laboratório de Cultura de Tecidos e Plantas Medicinais da UFLA. A propagação de $H$. marrubioides foi feita também em cultivo in vitro, em bandejas contendo substrato Plantmax ${ }^{\circledast}$, onde permaneceram por 30 dias. Após este período, realizou-se o transplantio das mudas em 22 de agosto de 2007, para 100 vasos plásticos contendo $10 \mathrm{~L}$ de composto orgânico. A colheita de folhas in natura foi realizada 275 dias após o transplantio das mudas, próximo às 7:00 h da manhã. A metodologia de coleta foi a mesma utilizada no ambiente em campo. Na época de colheita das folhas, as plantas estavam no estádio reprodutivo. 
Tabela 1. Dados climatológicos em Lavras, MG, no dia de coleta das folhas para o ambiente campo e casa de vegetação

\begin{tabular}{lcccccc}
\hline Dia de coleta & máx & $\begin{array}{c}\text { Temperatura }\left({ }^{\circ} \mathrm{C}\right) \\
\text { min }\end{array}$ & méd & $\begin{array}{c}\text { Precipitação total } \\
(\mathrm{mm})\end{array}$ & $\begin{array}{c}\text { UR* } \\
(\%)\end{array}$ & $\begin{array}{c}\text { Insolação } \\
(\text { horas })\end{array}$ \\
\hline CAMPO & 26,4 & 12,2 & 17,8 & 0 & 76,5 & 9,1 \\
CASA DE VEGETAÇÃO & 32 & 18 & 24 & 0 & 53,5 & 9,1 \\
\hline
\end{tabular}

*UR: umidade relativa

\section{Extração do óleo volátil}

Amostras compostas de $40 \mathrm{~g}$ de massa fresca das folhas oriundas de ambos ambientes de cultivo foram utilizadas para extração do óleo volátil pelo método de hidrodestilação em aparelho de Clevenger modificado, por $120 \mathrm{~min}$.

A mistura resultante de água e óleo volátil foi coletada e o óleo foi extraído com diclorometano $(3 \times 5 \mathrm{~mL})$. As frações orgânicas foram reunidas e secas com sulfato de magnésio anidro $(3,0 \mathrm{~g})$, deixando-o agir por $30 \mathrm{~min}$. Posteriormente, o sal foi removido por filtração simples e o solvente foi evaporado à temperatura ambiente sob capela de exaustão de gases. O teor percentual do óleo volátil foi calculado através da fórmula: T\% = Massa do óleo (g) / 40 g x 100.

$\mathrm{O}$ delineamento experimental foi inteiramente casualizado, com 10 repetições para cada ambiente de cultivo. As variáveis analisadas foram submetidas à análise de variância, ao nível de 5\% de probabilidade pelo teste de F. As análises estatísticas foram realizadas utilizando-se o software Sisvar ${ }^{\circledR}$, versão 5.0. ${ }^{12}$

\section{Composição química do óleo volátil}

As análises de composição química foram realizadas empregando uma amostra composta, compreendida da reunião de alíquotas equivolumétricas do óleo volátil das repetições de cada tratamento.

As análises foram realizadas utilizando um sistema de cromatografia em fase gasosa acoplada a um espectrômetro quadrupolar de massas (CG-EM), Shimadzu ${ }^{\circledR}$ QP5050A (Kyoto, Japão), nas seguintes condições operacionais: coluna capilar de sílica fundida, modelo CBP-5 (30 m de comprimento $\mathrm{x} 0,25 \mathrm{~mm}$ de d.i. $\mathrm{x} 0,25 \mu \mathrm{m}$ de espessura do filme em $5 \%$ de fenilmetilpolissiloxano) (Shimadzu ${ }^{\circledR}, J^{2}$ ão), hélio como gás de arraste com fluxo de 1,0 $\mathrm{mL} / \mathrm{min}$; aquecimento com temperatura programada $\left(60^{\circ} \mathrm{C}\right.$ com um gradiente de $3^{\circ} \mathrm{C} / \mathrm{min}$ até $240^{\circ} \mathrm{C}$ e, em seguida, com um gradiente de $10^{\circ} \mathrm{C} / \mathrm{min}$ até $270{ }^{\circ} \mathrm{C}$, mantendo-se uma isoterma de $7 \mathrm{~min}$, compreendendo um tempo total de corrida de $70 \mathrm{~min}$ ). A energia de ionização do detector foi de $70 \mathrm{eV}$, sendo o volume de injeção da amostra de 0,5 $\mu \mathrm{L}$ diluída em diclorometano (grau ultra-resíduo, Baker, EUA) e uma razão de injeção de 1:20. As temperaturas do injetor e do detector foram mantidas em 220 e $240{ }^{\circ} \mathrm{C}$, respectivamente. A análise foi conduzida no modo varredura, a uma velocidade de $1,0 \mathrm{scan} / \mathrm{s}$, com um intervalo de aquisição de massas de $40-400 \mathrm{~m} / \mathrm{z}$. A análise quantitativa foi obtida pela integração do cromatograma total de íons (TIC). A identificação dos constituintes foi realizada por comparação dos espectros de massas com o banco de dados das bibliotecas NIST/EPA/NHI, ${ }^{13}$ por comparação dos espectros de massas e índices de retenção (IR) com os da literatura, ${ }^{14} \mathrm{e}$ coinjeção com padrões autênticos. Os IR foram calculados através da coinjeção com uma mistura de hidrocarbonetos, $\mathrm{C}_{8}-\mathrm{C}_{32}$ (Sigma, EUA), e com aplicação da Equação de van Den Dool e Kratz. ${ }^{15}$

\section{RESULTADOS E DISCUSSÃO}

O óleo volátil de $H$. marrubioides, cultivada no campo, apresentou coloração levemente amarelada e intenso odor, enquanto o óleo obtido de plantas cultivadas em casa de vegetação se mostrou quase incolor e com odor menos acentuado.
De acordo com o teste de $\mathrm{F}$ houve diferença significativa no teor de óleo volátil de $H$. marrubioides para os ambientes de cultivo. Observou-se que plantas cultivadas em campo apresentaram maior teor de óleo volátil $(0,13 \%)$, sendo em média três vezes maior que no cultivo em casa de vegetação $(0,04 \%)$. Pelas análises de CG/EM foi possível identificar $99,19 \%$ dos constituintes químicos presentes no óleo volátil de plantas de $H$. marrubioides cultivadas no campo e $94,92 \%$ das cultivadas em casa de vegetação (Tabela 2).

A produção de óleo volátil é altamente influenciada por fatores genéticos, ecofisiológicos e ambientais. ${ }^{16} \mathrm{O}$ alto teor de óleo volátil encontrado em folhas de plantas de $H$. marrubioides cultivadas no campo pode ser atribuído às condições ambientais a que estas plantas foram submetidas. No cultivo em casa de vegetação, as plantas foram cultivadas em vasos, o que pode ter limitado o desenvolvimento e crescimento do sistema radicular. No campo, o sistema radicular tem maiores condições de desenvolvimento, devido a maior possibilidade de exploração do solo em busca de nutrientes. Outro fator que pode ter influenciado a obtenção de maior teor de óleo volátil nas plantas cultivadas no campo é a intensidade luminosa, que foi três vezes superior quando comparada ao sistema protegido, no entanto, estudos específicos de intensidade luminosa necessitam ser realizados.

No cultivo de duas espécies de manjericão (Ocimum minimum L. e Ocimum basilicum L.), em hidroponia e com diferentes substratos sob ambiente protegido, o método de cultivo não influenciou significativamente no rendimento e composição química dos óleos voláteis, evidenciando a regulação genética da biossíntese dessas substâncias. ${ }^{17}$ Resultados diferentes foram observados para $H$. marrubioides, para a qual o teor e a composição química do óleo volátil variaram com o sistema de cultivo empregado (casa de vegetação e campo), refletindo o dinamismo das interconversões que ocorrem continuamente entre os constituintes dos óleos voláteis, influenciadas por fatores ambientais. ${ }^{18-20}$

Os dados obtidos das determinações qualitativas e quantitativas dos componentes presentes no óleo volátil de $H$. marrubioides estão apresentados na Tabela 2.

Os constituintes químicos majoritários do óleo volátil das plantas cultivadas no campo foram trans-tujona $(9,98 \%), \beta$-cariofileno $(10,42 \%), \gamma$-muuroleno $(12,83 \%)$ e germacra-4(15),5,10(14)-trien-1$\alpha$-ol $(16,34 \%)$, correspondendo a $49,57 \%$ da composição química do óleo volátil (Tabela 2). A porcentagem desses mesmos constituintes químicos do óleo volátil obtido das plantas cultivadas em casa de vegetação correspondeu a $21,65 \%$, ou seja, 2,3 vezes menor que no campo, demonstrando as diferenças quantitativas desses óleos em função do sistema de cultivo empregado.

Os constituintes químicos $\alpha$-copaeno [5,27\% (campo), 5,83\% (casa de vegetação)], $\beta$-cariofileno $(10,42 ; 8,93 \%), \gamma$-muuroleno $(12,83 ; 11,58 \%)$ e óxido de cariofileno $(7,36 ; 8,03 \%)$ apresentaram porcentagens similares em ambos ambientes de cultivo (Figura 1a). De maneira geral, as porcentagens dos constituintes químicos identificados nos óleos voláteis foram maiores no cultivo em campo, com exceção de alguns constituintes como cariofila-4(14),8(15)-dien-5 $\beta$-ol $(4,14 ; 7,17 \%)$, cuja porcentagem foi maior no cultivo realizado em casa de vegetação (Figura $1 \mathrm{~A})$. 
Tabela 2. Porcentagem dos constituintes químicos do óleo volátil de H. marrubioides obtido de plantas cultivadas em campo e em casa de vegetação

\begin{tabular}{|c|c|c|c|c|}
\hline \multirow{2}{*}{$\mathrm{N}^{\circ}$} & \multirow{2}{*}{$\mathrm{IK}^{\mathrm{a}}$} & \multirow{2}{*}{ Constituinte } & \multicolumn{2}{|c|}{ Condição de cultivo } \\
\hline & & & Campo & Casa de vegetação \\
\hline 1 & 1098 & Linalol $^{\text {b }}$ & 0,58 & - \\
\hline 2 & 1104 & cis-Tujona & 3,36 & - \\
\hline 3 & 1114 & trans-Tujona & 9,98 & 0,41 \\
\hline 4 & 1132 & iso-3-Tujanol & 2,46 & $\mathrm{t}$ \\
\hline 5 & 1142 & cis-Verbenol & 1,31 & - \\
\hline 6 & 1143 & trans-Sabinol & - & 0,59 \\
\hline 7 & 1163 & 3-Tujanol & - & 0,64 \\
\hline 8 & 1171 & cis-3-Pinocanfona & 2,64 & $\mathrm{t}$ \\
\hline 9 & 1175 & Terpinen-4-ol & 1,12 & - \\
\hline 10 & 1188 & $\alpha$-Terpineol & 1,40 & 0,71 \\
\hline 11 & 1375 & $\alpha$-Copaeno & 5,27 & 5,83 \\
\hline 12 & 1387 & $\beta$-Bourboneno & 1,63 & - \\
\hline 13 & 1419 & $\beta$-Cariofileno ${ }^{\mathrm{b}}$ & 10,42 & 8,93 \\
\hline 14 & 1453 & $\alpha$-Humuleno & 0,86 & 1,01 \\
\hline 15 & 1480 & $\gamma$-Muuroleno & 12,83 & 11,58 \\
\hline 16 & 1483 & $\gamma$-Himachaleno & 3,54 & - \\
\hline 17 & 1491 & trans-Muurola-4(14),5-dieno & 0,10 & 1,30 \\
\hline 18 & 1514 & $\gamma$-Cadineno & 1,58 & 3,15 \\
\hline 19 & 1522 & $\delta$-Cadineno & 0,66 & 2,24 \\
\hline 20 & 1549 & Epóxido de italiceno & 1,07 & - \\
\hline 21 & 1550 & 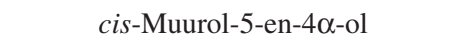 & - & 10,84 \\
\hline 22 & 1550 & Ocidentalol & 0,64 & 1,16 \\
\hline 23 & 1554 & $\mathrm{MM}=220^{\mathrm{c}}$ & 0,55 & 3,88 \\
\hline 24 & 1561 & (E)-Nerolidol & - & 1,45 \\
\hline 25 & 1581 & Óxido de cariofileno $^{\mathrm{b}}$ & 7,36 & 8,03 \\
\hline 26 & 1590 & $\beta$-Copaen- $4 \alpha$-ol & 0,97 & - \\
\hline 27 & 1591 & Salvial-4(14)-en-1-ona & 0,10 & - \\
\hline 28 & 1600 & Rosifoliol & - & 1,19 \\
\hline 29 & 1630 & Muurola-4,10(14)-dien-1 $\beta$-ol & 1,31 & 2,00 \\
\hline 30 & 1637 & Cariofila-4(14),8(15)-dien-5 $\alpha$-ol & 1,69 & 2,10 \\
\hline 31 & 1640 & Cariofila-4(14),8(15)-dien-5 $\beta$-ol & 4,14 & 7,17 \\
\hline 32 & 1645 & $\alpha$-Muurolol & 0,97 & 3,21 \\
\hline 33 & 1649 & $\beta$-Eudesmol & 0,67 & - \\
\hline 34 & 1652 & $\alpha$-Cadinol & - & 3,06 \\
\hline 35 & 1656 & $\mathrm{M}=220^{\mathrm{d}}$ & 2,15 & 3,01 \\
\hline 36 & 1660 & cis-Calamenen-10-ol & 0,60 & 0,79 \\
\hline 37 & 1669 & 14-Hidróxi-(Z)-cariofileno & 0,89 & - \\
\hline 38 & 1670 & Bulnesol & - & 1,43 \\
\hline 39 & 1680 & Kusinol & - & 1,66 \\
\hline 40 & 1684 & Germacra-4(15),5,10(14)-trien-1 $\alpha$-ol & 16,34 & 0,73 \\
\hline 41 & 1688 & Eudesma-4(15),7-dien-1 $\beta$-ol & - & 6,82 \\
\hline \multicolumn{3}{|c|}{ Total identificado } & 99,19 & 94,92 \\
\hline
\end{tabular}

aÍndice de Kovats experimental; b coinjeção com padrão; ' $\mathrm{IK}=1554, \mathrm{~m} / z$ (rel.int.): 220[M+1 $(10), 205(8), 202(5), 187(7), 163(13), 138(62), 123(47), 96(80)$, 79(87), 69(55), 55(50), 41(100); ${ }^{\mathrm{d}} \mathrm{IK}=1656, \mathrm{~m} / \mathrm{z}$ (rel.int.): 220[M+1 (38), 205(13), 204(20), 202(15), 187(12), 177(50), 159(100), 149(25), 131(31), 117(42), 105(50), 91(62), 81(64), 67(47), 55(37), 43(65), 41(52); -: constituinte não detectado; t: traço $(<0,05 \%)$. 
A)

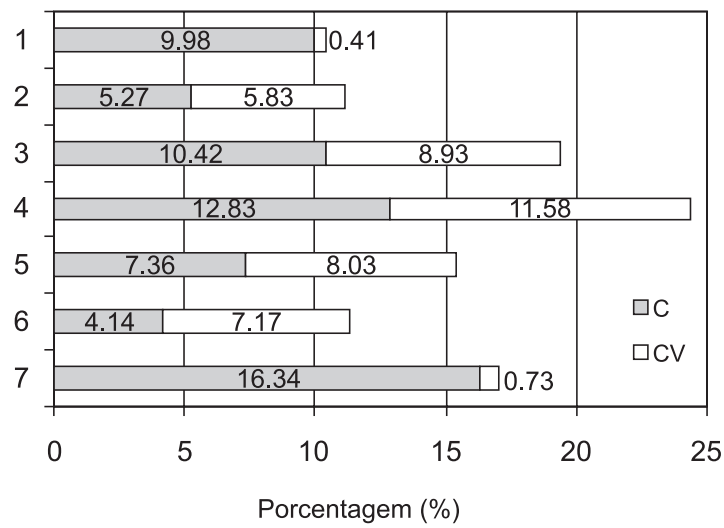

B)

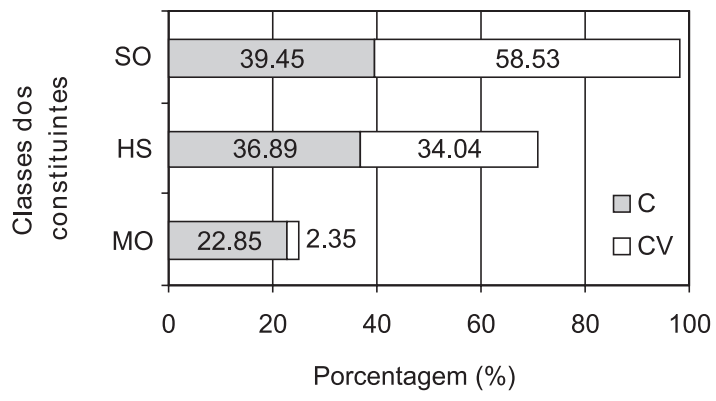

(A) 1, trans-tujona; 2, -copaeno; 3, -cariofileno; 4, -muuroleno; 5, óxido de cariofileno; 6, cariofila-4(14),8(15)-dien-5--ol; 7, germacra-4(15),5,10(14)-trien-1-ol. (B) SO, Sesquiterpenos Oxigenados; HS, Hidrocarbonetos Sesquiterpênicos; MO, Monoterpenos Oxigenados

Figura 1. Porcentagem correspondente aos compostos majoritários (A) $e$ às classes dos constituintes (B) identificados no óleo volátil em folhas de $H$. marrubioides no cultivo em campo $(C)$ e em casa de vegetação $(\mathrm{CV})$

Os principais constituintes químicos observados no óleo volátil extraído de folhas de plantas nativas de $H$. marrubioides coletadas em Lavras, MG, foram $\delta$-cadineno $(5,3 \%)$, epóxido de italiceno $(8,8 \%)$, óxido de cariofileno $(7,7 \%)$, guaiol $(6,2 \%)$, cariofila-4(14),8(15)-dien$5 \beta$-ol $(16,4 \%)$, 14-hidróxi-9-epi- $(E)$-cariofileno $(6,6 \%)$ e eudesma4(15),7-dien-1 $\beta$-ol (12,8\%). ${ }^{8}$ Diante desses dados, pode-se observar a existência de diferenças na composição química do óleo volátil de $H$. marrubioides determinada no presente trabalho e naquele analisado por Sales e colaboradores. Tal diferença provavelmente está relacionada com a origem do material vegetal, pois Sales e colaboradores trabalharam com plantas nativas, enquanto no presente estudo as plantas de $H$. marrubiodes foram cultivadas. A distinção na composição química desses óleos pode estar associada aos ambientes de cultivo, uma vez que as plantas nativas sofrem consequências adversas acentuadas, quando comparadas com plantas cultivadas, pois essas últimas não estão expostas a condições desfavoráveis como competição de espécies, estresses hídrico e de intensidades luminosas.

Diferenças qualitativas expressivas foram também observadas para alguns constituintes químicos, como a presença de cis-muurol5 -en-4 $\alpha$-ol, correspondendo a uma porcentagem de $10,84 \%$ da composição química do óleo volátil obtido do cultivo em casa de vegetação, ausente no cultivo em campo. Além desse constituinte, foi identificado a presença de trans-sabinol (0,59\%), 3-tujanol $(0,64 \%)$, $E$-nerolidol (1,45\%), rosifoliol (1,19\%), $\alpha$-cadinol (3,06\%), bulnesol $(1,43 \%)$, kusinol $(1,66 \%)$ e eudesma-4(15),7-dien-1 $\beta$-ol $(6,82 \%)$, perfazendo $27,68 \%$ da composição química do óleo volátil obtido das plantas oriundas de casa de vegetação, ausentes no óleo volátil obtido das plantas cultivadas no campo.
De maneira semelhante foi identificada a presença de linalol $(0,58 \%)$, cis-tujona $(3,36 \%)$, cis-verbenol $(1,31 \%)$, terpinen-4-ol $(1,12 \%), \beta$-bourboneno (1,63\%), $\gamma$-himachaleno (3,54\%), epóxido de italiceno $(1,07 \%)$, $\beta$-copaen- $4 \alpha$-ol $(0,97 \%)$, salvial-4(14)-en-1-ona $(0,10 \%)$, $\beta$-eudesmol $(0,67 \%)$ e 14-hidróxi-(Z)-cariofileno $(0,89 \%)$, totalizando $15,24 \%$ da composição química do óleo volátil obtido das plantas cultivadas no campo, ausentes no óleo volátil extraído das plantas oriundas da casa de vegetação. Diferenças qualitativas na composição do óleo volátil também foram observadas em plantas nativas e cultivadas de Baccharis trimera (Less.) DC. ${ }^{20}$

Houve uma diferença marcante entre os teores totais de monoterpenos e sesquiterpenos dos óleos voláteis obtidos de folhas de $H$. marrubioides no presente estudo para ambos sistemas de cultivo (Figura 1B). O teor de sesquiterpenos oxigenados foi maior nas plantas cultivadas em casa de vegetação $(58,53 \%)$, enquanto que os hidrocarbonetos sesquiterpênicos foram ligeiramente mais abundantes nas plantas cultivadas em campo (36,89\%). No entanto, a alteração mais expressiva na composição química do óleo volátil foi observada no teor de monoterpenos oxigenados, onde a porcentagem total de constituintes no óleo volátil extraído das folhas de plantas cultivadas no campo foi de $22,85 \%$, enquanto que daquele obtido das plantas cultivadas em casa de vegetação foi de apenas 2,35\% (Figura 1B).

Os monoterpenos são os principais componentes do óleo da família Lamiaceae. ${ }^{21,22}$ Resultados diferentes foram obtidos para $H$. marrubioides, em cuja composição química prevalecem constituintes pertencentes a classe dos sesquiterpenos.

As diferenças observadas nos teores e composições químicas dos óleos voláteis oriundos de cultivo em casa de vegetação e campo demonstram a necessidade de padronização de práticas de manejo agrícola, colheita e beneficiamento da matéria-prima vegetal em benefícios da qualidade e constância do produto final.

\section{CONCLUSÕES}

Foi verificada a interferência de técnicas de cultivo no teor e na composição química do óleo volátil em plantas de $H$. marrubioides. $\mathrm{O}$ maior teor do óleo volátil foi observado nas plantas cultivadas no campo. A composição química do óleo volátil apresentou diferenças marcantes entre a concentração de mono e sesquiterpenos nas plantas cultivadas em campo e casa de vegetação. A classe dos sesquiterpenos foi três vezes superior à dos monoterpenos nas plantas cultivadas em campo, enquanto que em casa de vegetação a concentração de sesquiterpenos foi trinta e nove vezes superior à dos monoterpenos. O constituinte químico majoritário do óleo das plantas de campo foi o germacra-4(15),5,10(14)trien- $1 \alpha$-ol e das plantas de casa de vegetação o $\gamma$-muuroleno.

Essa explícita diferença revela que o ambiente de cultivo é um fator importante para o teor e a composição química do óleo volátil de plantas de $H$. marrubioides, demonstrando a forte relação entre essas variáveis.

\section{AGRADECIMENTOS}

À Coordenação de Aperfeiçoamento de Pessoal de Nível Superior (CAPES) pela bolsa de estudo de P. P. Botrel. Ao Conselho Nacional de Desenvolvimento Cientifico e Tecnológico (CNPq) pelas bolsas de produtividade (J. E. B. P. Pinto e P. H. Ferri). À Fundação de Amparo à Pesquisa de Minas Gerais (FAPEMIG) e ao CNPq pelo apoio financeiro do projeto.

\section{REFERÊNCIAS}

1. Harley, R. M. Em Evolution and distribution of Eriope (Labiatae) and its relatives in Brasil; Vanzolini, P. E.; Heyer, W. R., eds.; Academia 
Brasileira de Ciências: Rio de Janeiro, 1988.

2. Corrêa, M. P.; Dicionário das Plantas Úteis e das Exóticas Cultivadas, Imprensa Nacional: Rio de Janeiro, 1931.

3. De Oliveira, C. M. A.; Silva, M. D. R.; Kato, L.; Da Silva, C. C.; Ferreira, H. D.; Souza, L. K. H.; J. Braz. Chem. Soc. 2004, 15, 756.

4. Souza, L. K. H.; Oliveira, C. M. A.; Ferri, P. H.; Oliveira, J. G.; Souza, A. H.; Fernandes, O. D. L.; Silva, M. D. R.; Mem. I. Oswaldo Cruz. 2003, 98, 963.

5. Barbosa, P. P. P.; Ramos, C. P.; Phytother Res. 1992, 6, 114.

6. Costa, J. G. M.; Rodrigues, F. F. G.; Angélico, E. C.; Silva, M. R.; Mota, M. L.; Santos, N. K. A.; Cardoso, A. L. H.; Lemos, T. L. G.; Rev. Bras. Farmacogn. 2005, 15, 304.

7. Bueno, A. X.; Moreira, A. T. S.; Silva, F. T.; Estevam C. S.; Marchioro, M.; Rev. Bras. Farmacogn. 2006, 16, 317.

8. Sales, J. F.; Pinto, J. E. B. P.; Botrel, P. P.; Oliveira, C. B. A.; Ferri, P. H.; Paula, J. R.; Seraphin, J. C.; J. Essential Oils Res. 2007, 19, 552.

9. Castro, D. M.; Ming, L. C.; Marques, M. O. M.; Revista Brasileira de Plantas Medicinais 2002, 4, 75 .

10. Corrêa Júnior, C.; Scheffer, M. C.; Ming, L. C; Cultivo agroecológico de plantas medicinais, aromáticas e condimentares, Ministério do Desenvolvimento Agrário: Brasília, 2006.

11. Simões, C. M. O.; Spitzer, V.; Farmacognosia, $5^{\text {a }}$ ed., UFRGS: Porto Alegre, 2000.
12. Ferreira, D. F.; Anais da $45^{\circ}$ Reunião Anual da Região Brasileira da Sociedade Internacional de Biometria, São Carlos, Brasil, 2000.

13. National Institute of Standards and Technology; PC version of the NIST/ EPA/NIH Mass Spectral Databas, Gaithersburg: USA, 1998.

14. Adams, R. P.; Identification of essential oil components by gas chromatography/mass spectrometry, $4^{\text {th }}$ ed. Allured Publishing Corporation: Illinois, 2007.

15. van den Dool, H.; Kratz, P. D.; J. Chromatogr. 1963, 11, 463.

16. Sangwan, N. S.; Farooqi, A. H. A.; Shabih, F.; Sangwan, R. S.; Plant growth regul. 2001, 34, 21.

17. Fernandes, P. C.; Facanali, R.; Marques, M. O. M.; Teixeira, J. P. F.; Furlani, P. R.; Horticultura Brasileira 2004, 22, 260.

18. Santos, M. R. A.; Innecco, R.; Horticultura Brasileira 2004, 22, 182.

19. Castro, D. M.; Tese de Doutorado, Universidade Estadual Paulista, Brasil, 2001.

20. Silva, F. G.; Oliveira, C. B. A.; Pinto, J. E. B. P.; Nascimento, V. E.; Santos, S. C.; Seraphin, J. C.; Ferri, P. H.; J. Braz. Chem. Soc. 2007, 18, 997.

21. Phatak, S.; Heble, M. R.; Fitoterapia 2002, 73, 32.

22. Scavroni, J.; Boaro, C. S. F.; Marques, M. O. M.; Ferreira, L. C.; Brazilian J. Plant Physiology 2005, 17, 345. 